\title{
High field septum magnet using a superconducting shield for the Future Circular Collider
}

\begin{abstract}
Dániel Barna*
Wigner Research Centre for Physics, Budapest H-1121, Hungary

(Received 15 July 2016; revised manuscript received 16 February 2017; published 21 April 2017)

A zero-field cooled superconducting shield is proposed to realize a high-field (3-4 T) septum magnet for the Future Circular Collider hadron-hadron (FCC-hh) ring. Three planned prototypes using different materials and technical solutions are presented, which will be used to evaluate the feasibility of this idea as a part of the FCC study. The numerical simulation methods are described to calculate the field patterns around such a shield. A specific excitation current configuration is presented that maintains a fairly homogeneous field outside of a rectangular shield in a wide range of field levels from 0 to 3 Tesla. It is shown that a massless septum configuration (with an opening in the shield) is also possible and gives satisfactory field quality with realistic superconducting material properties.
\end{abstract}

DOI: 10.1103/PhysRevAccelBeams.20.041002

\section{INTRODUCTION}

In its early phase of design the parameters of the Future Circular Collider's hadron-hadron ring (FCC-hh) are subject to frequent and quite significant changes. Table I shows two sets of parameters of the injection/extraction system of the FCC [shown schematically in Fig. 1(a)] compared to the LHC. In the May'16 parameter set the extraction straight section was $3.5 \mathrm{~km}$, but a matching quadrupole placed $90 \mathrm{~m}$ downstream of the septa required a strong septum magnet to reach the clearance necessary to avoid the magnet's cryostat. After a recent redesign of the optics (Jan'17 parameters) the extraction straight section has a shorter length of $2.8 \mathrm{~km}$ but $300 \mathrm{~m}$ drift space downstream of the septa, which allows for a weaker septum magnet. The May'16 parameter set triggered concerns about the applicability of the existing technologies (for example the Lambertson septum magnets of the LHC with about $1 \mathrm{~T}$ field) for the FCC. Scaling this technology in length would have led to $6 \mathrm{MW}$ power dissipation for the two beams, and a beam line of over $500 \mathrm{~m}$ including pumps, gauges, etc., largely exceeding the available space. Even though more space could have been allocated for the septum section, and the most recent parameter set releases the requirements on the septa, a more compact solution is highly desirable and could find applications in other facilities as well. The necessary increase of the field of the magnet to above $2 \mathrm{~T}$ requires superconducting coils to keep the power consumption acceptable. At such high fields the saturation of the iron yoke and the resulting leakage field to the

\footnotetext{
*barna.daniel@wigner.mta.hu

Published by the American Physical Society under the terms of the Creative Commons Attribution 3.0 License. Further distribution of this work must maintain attribution to the author(s) and the published article's title, journal citation, and DOI.
}

circulating beam require further changes of the topology [1]. In order to be ready for a safety beam abort at any time, the septum magnet must follow the energy of the ring, i.e. operate in slow-ramp or quasi-DC mode. In the high-field region the magnetic field must be homogeneous over the transverse range that the deflected beam will cover, in order to avoid complicated tracking studies and position sensitivity. The coordinated R\&D activity associated with the FCC project provides an ideal environment for the development of novel concepts and technologies addressing these challenges.

\section{SUPERCONDUCTING SHIELD}

A field-free region within a magnetic field (such as that needed for a septum magnet) can be realized using superconductors relying on two effects: (a) The Meissner effect which is of little practical use for this purpose since its critical field is typically a few-10 mT. (b) The induction of persistent shielding currents on the surface of the material in a ramped magnetic field, which oppose any change of the field within the material.

A superconducting shield installed around the circulating beam and cooled below the critical temperature $T_{c}$ in zero field will therefore "freeze" the zero field at its interior, as long as the shield thickness is large enough for a given external field strength and the lifetime of the shielding currents is sufficiently long. The time scale for this last criterion is set by the length of a cycle of the FCC (about 10 hours for the LHC). The principle is similar to that used by pulsed eddy current septa except that the shielding currents do not decay, and the device can be used in slowramp or quasi-DC mode as required. The principle is illustrated in Fig. 1(b). The idea to create a field-free region within a strong magnetic field using superconductors is not new [2-7], but it has not yet been applied to create an accelerator septum magnet (the shield applied in 
TABLE I. Parameters of the LHC and FCC injection and extraction systems at the time of writing this article (subject to frequent changes in case of the FCC study).

\begin{tabular}{|c|c|c|c|c|c|}
\hline & \multicolumn{2}{|c|}{ LHC } & \multicolumn{3}{|c|}{ FCC-hh } \\
\hline & Injection & Extraction & Injection & Extraction (May.'16) & Extraction (Jan.'17) \\
\hline Beam energy $[\mathrm{TeV}]$ & 0.45 & $0.45-7$ & 1.3 or 3.3 & $3.3-$ & -50 \\
\hline Stored top beam energy [GJ] & & 0.36 & & 8. & \\
\hline Leak field to circulating beam (relative) & & $0^{-5}$ & $1.25 \times 10^{-4}$ & $1.25 \times$ & $10^{-4}$ \\
\hline Field homogeneity [\%] & & & 0.5 & 1 & \\
\hline Apparent septum thickness [T in Fig. $4(\mathrm{~b})][\mathrm{mm}]$ & & & & 2 & \\
\hline Extraction straight section length $[\mathrm{km}]$ & & & & 3.5 & 2.8 \\
\hline Deflection by septum $[\mathrm{mrad}]$ & 12 & 2.4 & 7.3 & 3.04 & 1.14 \\
\hline $\int B \mathrm{~d} l[\mathrm{Tm}]$ & 18 & 56 & 80.4 & $35-508$ & $12.5-190$ \\
\hline Available space [m] & 22 & 100 & 100 & 260 & 120 \\
\hline
\end{tabular}

[6] only shielded the leakage field of the injector magnet, and not the full field). The device will be referred to as a superconducting shield septum, or SuShi septum.

The principle has the following advantages. (i) The shielding currents are automatically arranged by nature, there is no need for precise coil windings to create zero field at the circulating beam. (ii) The continuous $2 \mathrm{D}$ shielding current distribution creates a perfect shield, in contrast to the discrete wires of magnets. (iii) According to the critical state model the shielding currents flow everywhere at the local critical current density $J_{c}(B)$, i.e. the highest possible value, giving the thinnest possible shield. (iv) The bulk superconductor shield has no windings with interleaving insulating materials, which worsen thermal and mechanical stability. This is advantageous especially close to the beams, where space is very tight and cooling and mechanical support is more difficult. The superconducting excitation coils with a more complicated structure are further away from the beams. (v) The individual turns of a magnet's coil are connected in series. The current in each turn is therefore equal and the current pattern is defined by the winding's geometry and cannot dynamically change. In case the superconducting cable becomes normal conducting locally, the current of that loop must be ramped down very quickly to avoid a fatal damage. This can only be done by ramping down the current of the whole coil which is difficult due to its large inductance. A quench heater is needed to quickly heat the whole coil above its critical temperature and thereby distribute the heat dissipation over the entire coil; propagation of the heat from the hot spot is not sufficient. On the contrary, the shielding currents in a passive shield can be treated as parallel current loops. During a magnetothermal runaway (flux jump) the current pattern can rearrange and the quickly changing magnetic field will trigger a transition of other parts of the shield as well to the normal-conducting state causing a
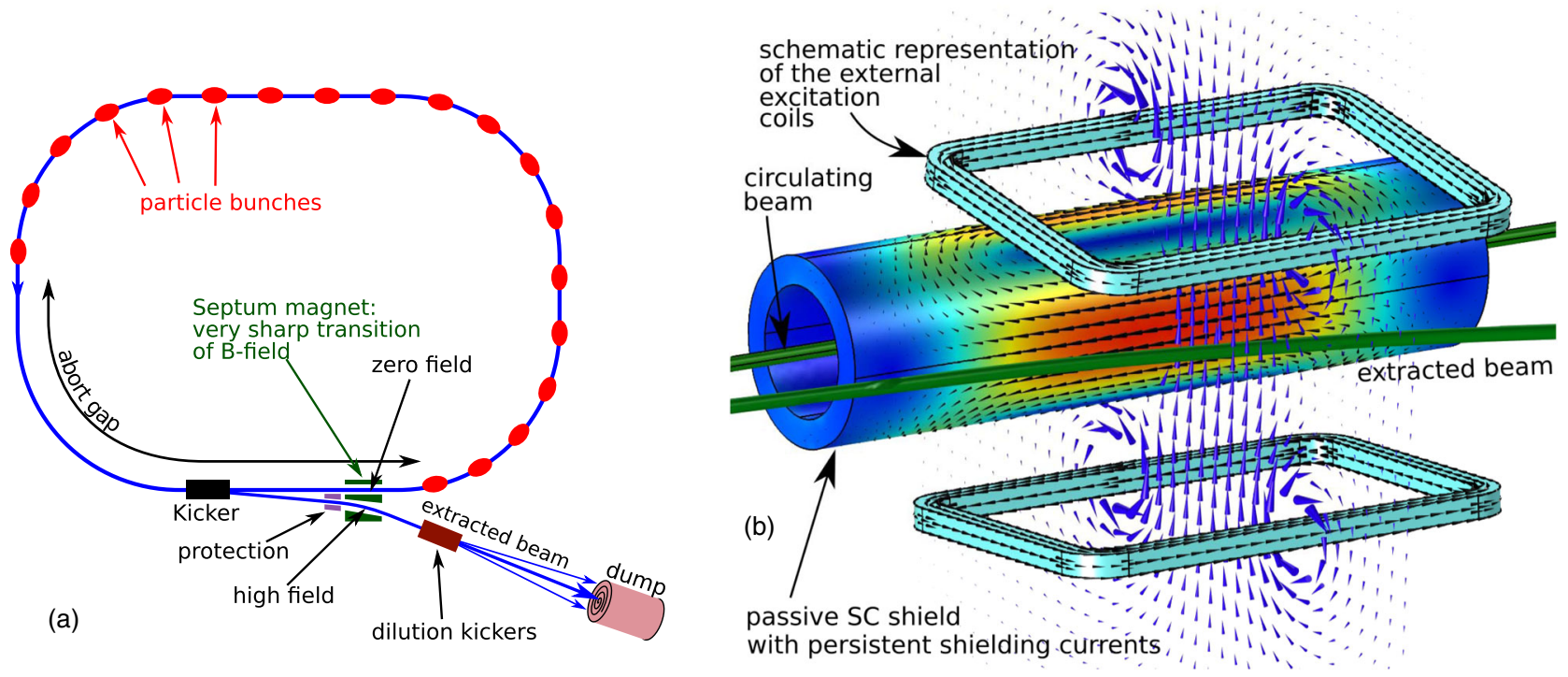

FIG. 1. (a) Layout of the beam extraction system of the FCC. (b) The principle of the superconducting shield. Blue arrows show the magnetic field in the transverse midplane; black arrows and color show the persistent shielding currents on the superconductor shield's surface. 
partial or full flux jump. A superconducting shield can survive a flux jump without additional heaters, further simplifying the structure of the septum blade. A flux jump could nevertheless inductively trigger a quench of the superconducting excitation coils, which will still need proper protection. These coils are further away from the beam where there is more space.

The disadvantages of this configuration are as follows. (i) The high radiation in the extraction zones can affect the reliability of the device, and lead to heating and quenches (this holds for all superconducting solutions). (ii) The shield is a passive device, i.e. it has no current leadsthis means that there is no "recovery" (i.e. restoration of the ideal state) in case of a thermal perturbation, even if the superconductor recovers thermally and undergoes a partial flux jump only. This means that the field configuration and the shield state are not a function of the controllable parameters $T$ and $B$ only, but also that of the history of the device. In case of field penetration a full "reset cycle" is needed to recover the device: heating above $T_{c}$ and cooling down in zero field.

There are two possible arrangements for beam extraction with a SuShi septum: the circulating beam is running within the shield in zero field, and the extracted beam is running in the high-field region of the device, or vice versa. In the following studies the first configuration will be assumed for the following reason. The quality of the field (i.e. the absence of higher order components) is more important at the circulating beam than at the extracted beam. If the shield is thick enough, it is easier to create a high-quality zero field inside the shield (the field is fully shielded inside the superconductor) than a high-quality strong dipole field outside the shield.

There is a wide range of applications of superconductors to shield [2-7] or shape [7-9] magnetic fields. Some unique challenges of the current application are as follows: (i) The bulk superconductor must fulfil these two functionalities simultaneously: completely shield the field at the location of the circulating beam, and shape a homogeneous field in a region of about a few $\mathrm{cm}$ outside the shield. (ii) The aimed magnetic field is rather high: 3-4 T. (iii) The field homogeneity must be independent of the field strength, spanning a range of a factor 15 between injection and ejection. (iv) The design must include a coupled optimization of the superconductor's shape and the external magnet's windings, rather than assume a given (for example homogeneous) external background field as in most applications.
Producing a homogeneous field outside the shield can be realized by the so-called magnetic cloak configuration [10] in an external dipole field, where a superconducting cylinder is surrounded by a ferromagnetic one, which absorbs the magnetic induction lines expelled by the superconducting tube. This configuration needs a thicker physical septum due to the presence of the ferromagnetic layer, it introduces yet another type of material into the cryogenic environment, and produces a very high concentration of magnetic field in the ferromagnetic material. The field enhancement factor $\left(d_{f}+R_{s}\right) / d_{f}$ is at least 5 for realistic parameters, where $d_{f}$ is the thickness of the ferromagnetic material, and $R_{s}$ is the outer radius of the superconducting shield. With the targeted field of 3-4 T this would give field levels exceeding $15-20 \mathrm{~T}$ in the ferromagnetic tube. This is considered as a prohibitive factor, and this configuration has therefore been dropped from the list of candidate technologies. Nevertheless, as a side remark, it must be noted that the saturation of the ferromagnetic material at higher field levels could eventually be compensated by the increasing penetration depth of the field into the superconducting shield.

In a possible scenario the extraction septum section of the FCC could be constructed from more robust normalconducting septa followed by the SuShi units in order to protect these latter ones from particle showers created upstream (Fig. 2). It is important to note here that a SuShi septum realizes a same-plane extraction scheme (kicker and septum acting in the same plane), in contrast to the Lambertson septa used in the LHC for example, restricting the possible normal-conducting and SuShi septum combinations. The SuShi septa would be classified into a few types installed at different locations along the extraction channel, according to the distance between the circulating and ejected beams, i.e. the shielded and homogeneous field regions. The SuShi septa would be a few meters long, depending on different design considerations. The superconducting shields must safely exceed the fringe field region of the external magnets in the longitudinal direction in order to avoid the leaking of stray fields to the circulating beam's position.

The studies presented later in this paper address the question of producing a homogeneous field outside the shield at both low and high field levels - a requirement of this specific application. Stability against flux jumps-a key point in shielding high magnetic fields by persistent shielding currents - will be assumed for the time being, based on the

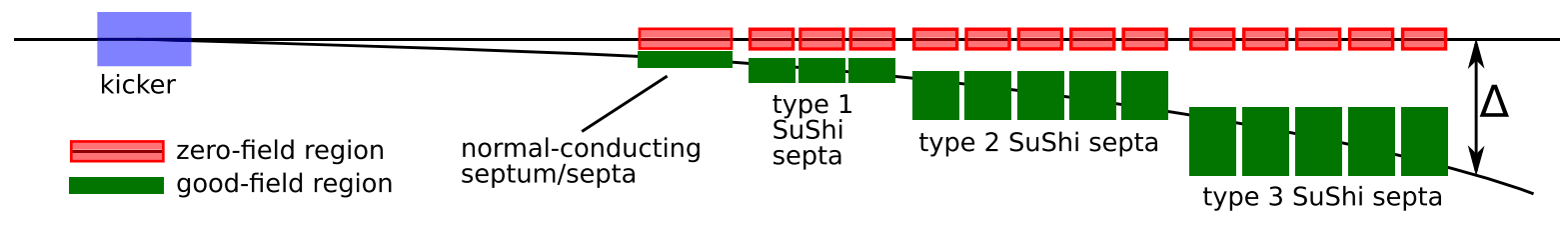

FIG. 2. Possible longitudinal layout of the extraction system. 
numerous successful projects documented in the literature $[11,12]$. Later studies and experimental tests should address this question as well in a realistic environment.

\section{POSSIBLE MATERIALS AND CONFIGURATIONS}

The $1 \mathrm{~mm}$ thick multilayer $\mathrm{NbTi} / \mathrm{Nb} / \mathrm{Cu}$ sheet (the $2 \mathrm{D}$ analogue of standard copper-stabilized multifilamentary NbTi superconducting cables) used by the BNL g-2 inflector [6,7] consists of 30 layers of $10 \mu \mathrm{m}$ thick $\mathrm{NbTi}$ sandwiched between $\mathrm{Nb}$ and $\mathrm{Cu}$ layers. Four stacked sheets (i.e. $4 \mathrm{~mm}$ total thickness) could shield a field of $3 \mathrm{~T}$ [7]. The allowed septum thickness could easily contain even more sheets to have a safety margin, embedded in a copper block [Fig. 3(a)] serving as a mechanical support and thermal stabilizer, including liquid-He cooling channels as well. The sheet is a discontinued commercial product of Nippon Steel Ltd. A semifinished $4.25 \mathrm{~mm} \times 410 \mathrm{~mm} \times$ $440 \mathrm{~mm}$ sheet has been purchased from their remaining stock and will be postprocessed into two pieces of $0.8 \mathrm{~mm} \times 195 \mathrm{~mm} \times 1000 \mathrm{~mm}$ sheets (rolling and heat treatment stages) by Kinzoku Oyo Giken Corp. in Japan. This material will then be used to manufacture two half cylinders of $25 \mathrm{~mm}$ radius and $450 \mathrm{~mm}$ length, each consisting of four layers, in a similar configuration as shown in [2].

Commercially available superconducting tapes (HTS, $\mathrm{Nb}_{3} \mathrm{Sn}, \mathrm{MgB}_{2}$ ) have a small width (10-15 mm) which is insufficient to make a jointless tube with a diameter of about $4 \mathrm{~cm}$ that does not intercept the path of the shielding currents. The technology developed at CERN to produce wide $\mathrm{Nb}_{3} \mathrm{Sn}$ tapes [5] did not make its way to the market, and seems to have disappeared. A superconducting shield could nevertheless be produced by helically winding and soft-soldering several layers of tape onto a supporting tube [Fig. 3(b)], each layer shifted by half the tape width. The mechanism of shielding is illustrated in [13]. The efficiency of this configuration is yet to be tested. These tapes have typically a minimum bending radius $(\sim 2.5 \mathrm{~cm})$ which is compatible with the parameters of a septum magnet.
A shield with a length of $450 \mathrm{~mm}$ and outer diameter of about $45 \mathrm{~mm}$ has been manufactured by SuperOx, Russia, for the time being consisting of 25 layers. If this configuration proves to be efficient at moderate fields $(\sim 1.5 \mathrm{~T})$, the shield will be upgraded to 50 layers, which is a very rough estimation to reach $3 \mathrm{~T}$ shielded field at its surface.

A third option is bulk $\mathrm{MgB}_{2}$, which can be produced either by the powder-in-tube (PIT) method, or by the reactive liquid $\mathrm{Mg}$ infiltration (RLI) process introduced by Giunchi [14]. With the PIT method $\mathrm{MgB}_{2}$ powder is filled between two concentric titanium tubes and a copper rod is inserted into the inner one to reinforce it. After compacting the powder the assembly is rolled to the shape shown in Fig. 3(c), and the central copper rod is drilled or etched away. A final heat treatment at $500-600^{\circ} \mathrm{C}$ fuses the grains of the powder. The RLI process starts with a central $\mathrm{Mg}$ rod and boron powder filled around it in a metallic container. Heat treatment at $T=850^{\circ} \mathrm{C}$ for a few hours melts the $\mathrm{Mg}$ which penetrates the boron preform and reacts with it. The final shape is the same as that shown in Fig. 3(c) (rectangular or cylindrical, as needed) without the inner Ti tube. RLI results in a material with slightly higher density and larger critical current density. Current technical limitations at Columbus Superconductor would have set an upper limit of $16 \mathrm{~mm}$ on the outer size of the shield manufactured by the PIT method. The RLI method can produce very large bulk pieces. A tube with a length of $450 \mathrm{~mm}$, outer diameter of $49 \mathrm{~mm}$ and wall thickness of $8 \mathrm{~mm}$ has been manufactured by E.P.C. S.R.L. (Italy) by the RLI process. Tests with all three candidate technologies mentioned above are planned in 2017.

\section{FIELD CALCULATION METHODS}

Since the length of the SuShi septum (along the $z$ coordinate) is much larger than its transverse size, a 2D simulation was done assuming $z$-invariance, $B$ being in the transverse plane $\left(B_{z}=0\right), A_{x}=A_{y}=0$ and induced shielding currents running along the length of the shield, i.e. $J_{x}=J_{y}=0$, where $B$ denotes the magnetic induction,
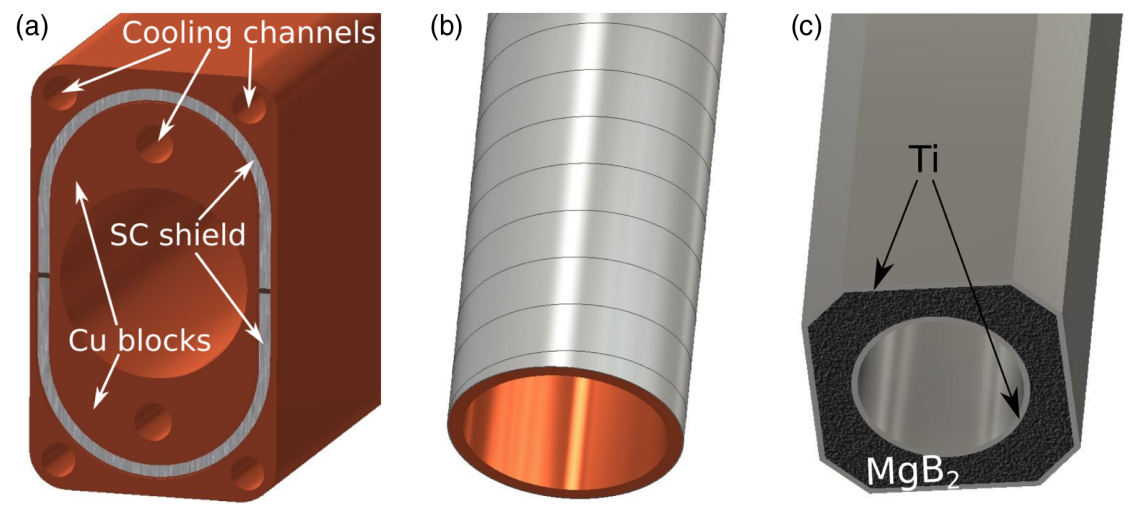

FIG. 3. Possible shield configurations with different materials. See text for details. 
$A$ is the magnetic vector potential and $J$ is the current density.

Neglecting the effects of flux creep and the decay of the shielding currents, the critical state of superconductors in an external field [15] is a steady state which is the result of a dynamic process of the currents being induced by the magnetic field. An often used method is to simulate this time-dependent phenomenon with a finite-element software, plugging in the highly nonlinear $E-J$ characteristics of the superconductor [a power-law dependence $E \propto$ $\left(J / J_{c}\right)^{n}$ for example with a very large exponent $\left.n\right]$. This is a very time-consuming method. When the external field is slowly ramped, the virgin magnetization of a superconductor can be directly simulated using the method described in [16], without the time-dependent process, which is therefore much faster and better suited for an optimization analysis. Its basic equation $\nabla \times\left(\nabla \times A_{z}\right)=$ $\mu_{0} J=-\operatorname{sign}\left(A_{z}\right) \mu_{0} J_{c}\left[1-\exp \left(-\left|A_{z} / A_{r}\right|\right)\right]$ relates the current density to the value of the vector potential $A_{z}$ and not its derivative, and hence becomes sensitive to the gauge choice. An offset of $A_{z}$ shifts the transition boundaries between zones of positive and negative shielding currents, and hence the integral of $J_{z}$ over the cross section of the shield. Since the superconducting shield does not have current leads, this integral must be zero. In order to satisfy this constraint the current density was defined as $J=-J_{c}(|B|) \tanh \left[\left(A_{z}-A_{0}\right) / A_{r}\right]$, where the original $\operatorname{sign}\left(A_{z}\right)\left[1-\exp \left(-\left|A_{z} / A_{r}\right|\right)\right]$ function was replaced by $\tanh \left(A_{z} / A_{r}\right)$ (following the comments of [17], although this particular function was not listed there), and the $A_{0}$ parameter was determined such that the integral of $J$ is zero. This parameter will then coincide with the value of the vector potential $A_{z}$ in the shielded region. The algorithm was implemented in the finite-element analysis software COMSOL.

Two materials were modeled by using their $J_{c}(B)$ curve in the simulation [Fig. 4(a)]: (a) the $\mathrm{NbTi} / \mathrm{Nb} / \mathrm{Cu}$ multilayer sheet at $4.2 \mathrm{~K}$ (material prepared by 120 hours heat treatment at $400^{\circ} \mathrm{C}$, with current paths parallel to the rolling direction of the sheet, open triangles of Fig. 2 in [18]), and (b) $\mathrm{MgB}_{2}$ at $T=20 \mathrm{~K}$ [19]. The cited papers show more performant $J_{c}(B)$ curves as well for both materials. This choice is therefore a conservative approach. For (a) the values of [18], reported for the superconducting NbTi layers only, were multiplied by the filling factor 0.36 to get the engineering current density of the real sheet. Due to its structure this material is nonisotropic, having a finite resistance in the direction perpendicular to its layers. This means that in a steady state the integral of the shielding currents should be equal to zero in each layer separately. Other states have currents running through the resistive copper layers, and will therefore relax towards a steady state probably slowly, since the resistive fraction of the current loops is very small. Evaluation and estimation of this effect is not attempted here; the material was modeled as an isotropic medium. $\mathrm{MgB}_{2}$ at $T=20 \mathrm{~K}$ has a very steep curve with 2 orders of magnitude difference between $B=0$ and $3 \mathrm{~T}$, which provides therefore a significantly different alternative to (a). Even though it is not very probable that the shield would be maintained at $T=20 \mathrm{~K}$ in an environment otherwise at $4.2 \mathrm{~K}$, this curve was used in the simulation as a "worst case" scenario of what can be done with $\mathrm{MgB}_{2}$. Since it is a bulk material with a filling factor of 1 , its engineering critical density is the same as the bulk critical current density. Since the shield constructed from an HTS tape has a more complicated 3D current pattern than the simpler bulk shields, its simulation was not attempted here.

\section{ANALYSIS AND RESULTS}

Figure 4(b) shows the schematic geometry of the superconducting shield and vacuum chambers. The thickness $d$ of the vacuum pipes is $1.5 \mathrm{~mm}$, including beam screens.

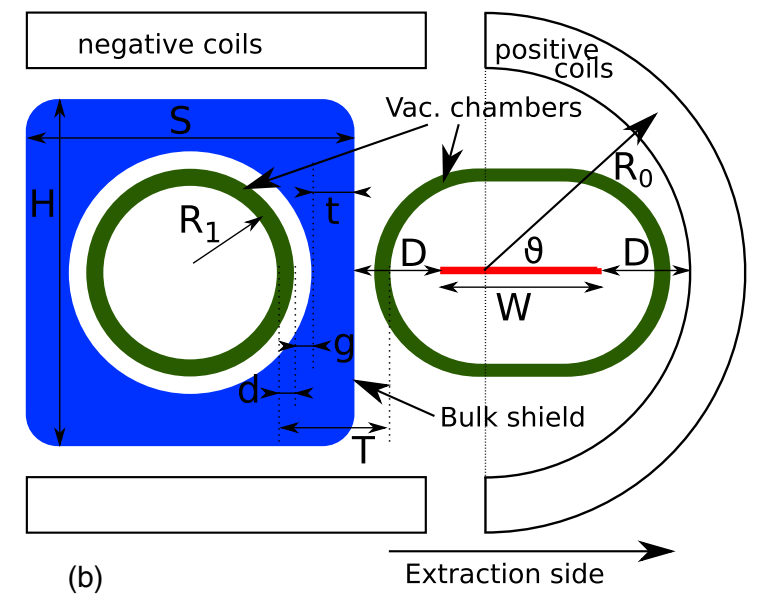

FIG. 4. (a) $J_{c}(B)$ curves used in the simulation for the NbTi/Nb/Cu multilayer sheet [18] and bulk $\mathrm{MgB}_{2}$ [19]. (b) Geometry of the shield, vacuum pipes and excitation coils. The thick red line indicates the required good field region. 
The highest allowed apparent septum thickness $T$ is $25 \mathrm{~mm}$. A shield thickness $t$ of $15 \mathrm{~mm}$ (possibly including nonsuperconducting materials as well, see Fig. 3) is sufficient to shield fields of 3-4 T, and leaves a gap $g=3.5 \mathrm{~mm}$ between the shield and vacuum chambers. This gap can be used for thermal decoupling between the vacuum chambers and the shield if needed, or for the cryogenic liquid if possible. These technical details are neglected in this conceptual study for the time being. The shield width $S$ (outer diameter in case of a cylindrical shield) was chosen to be $7 \mathrm{~cm}$, resulting in $R_{1}=17 \mathrm{~mm}$. The height $\mathrm{H}$ of the shield was slightly larger $(8 \mathrm{~cm})$ since this seemed to improve the homogeneity of the field.

Setting a target value of $B=3 \mathrm{~T}$ gives a beam trajectory radius of $56 \mathrm{~km}$ at $50 \mathrm{TeV}$. Using the May'16 parameters, the required deflection of $3 \mathrm{mrad}$ would lead to a transverse displacement of the beam in the septum section $(\Delta$, see Fig. 2) of at least $250 \mathrm{~mm}$ (ignoring gaps between the septa). Counting with five different types (only three types shown in Fig. 2) of SuShi septum magnets (with differing distance of the homogeneous field region from the circulating beam as we move downstream), transverse displacement in one class is about $50 \mathrm{~mm}$. The good field region must be $30 \mathrm{~mm}$ larger (Table I), giving $80 \mathrm{~mm}$. Presumably it will be much easier to create larger good field regions in the types installed more downstream, where the ejected beam is further away from the shield causing the fielddependent distortions. This allows a nonequal distribution of good field region widths among the different types as shown in Fig. 2. Therefore in the following analysis the homogeneity was required in a range of $W=50 \mathrm{~mm}$ only, starting at $D=15 \mathrm{~mm}$ away from the shield surface. With the values of the Jan'17 parameter set, the displacement $\Delta$ in the whole septum section is $36 \mathrm{~mm}$ only, which can almost be accommodated in the above-mentioned good field region with the $30 \mathrm{~mm}$ margin.

For simplicity, the excitation coils were modeled as zones with a simple geometrical shape [see Fig. 4(b)] with a thickness of $15 \mathrm{~mm}$ and a parametrized continuous current density distribution which was allowed to vary spatially. The extraction side of the device resembles one half of a dipole magnet, therefore a $\cos (\theta)$-like excitation coil was placed here with a current density parametrized as $J(r, \vartheta)=J_{p}[\cos (\vartheta)+\delta] \cdot R_{0} / r$ [where $r$ is the distance from the center of the positive coil, and see Fig. 4(b) for the explanation of the other variables]. The current density in the negative windings was taken as a piecewise linear function of the $x$ coordinate with six control values along the length of the block. The two current densities were normalized to give the same absolute total current. These windings can be realized by standard superconducting cables, with their spacing adjusted to approximate these current densities. The excitation current densities in the results reported below are less than $400 \mathrm{~A} / \mathrm{mm}^{2}$ everywhere, which is within the reachable limit by standard superconducting cables.

Figure 5 shows the field pattern around a rectangular $\mathrm{NbTi} / \mathrm{Nb} / \mathrm{Cu}$ shield for a current configuration that was found to give satisfactory results at field values of $B=0.5$ and $3 \mathrm{~T}$. The homogeneity $\Delta B_{y} / B_{y}$ was around $1 \%$ within the good field region at both field levels despite the fact that penetration into the superconducting shield is significantly different in the two cases. For $\mathrm{MgB}_{2}$ at $T=20 \mathrm{~K}$ a current configuration giving a homogeneity around $2 \%$ at both field levels was found (not shown). Figure 6 shows the shielding and excitation currents at $3 \mathrm{~T}$ for the two materials. Due to the steep $J_{c}(B)$ curve of $\mathrm{MgB}_{2}$ at $T=20 \mathrm{~K}$ [19] the shielding currents are concentrated along a front line where $|B|$ approaches zero.

Figure 7 shows details of these patterns in the horizontal midplane. Figure 7(a) shows $B_{y}$ for the two materials at two different field levels. Figure 7(b) zooms into the surface region of the shield, and displays the details of the attenuation of the magnetic field as a function of penetration depth, and the actual shielding currents $J(x)$ and the critical current density at the local value of the magnetic field $J_{c}[B(x)]$. Since for the square shield the derivatives with respect to the $y$ coordinate are much smaller than in the $x$ direction at the symmetry plane, Ampère's law can be well approximated as $\partial_{x} B_{y}=\mu_{0} J_{z}=\mu_{0} J_{c}\left(\left|B_{y}\right|\right)$. This is a one-dimensional ordinary differential equation which can be solved numerically $\left[B_{1 D}(x)\right]$ using the $J_{c}(B)$ curve of
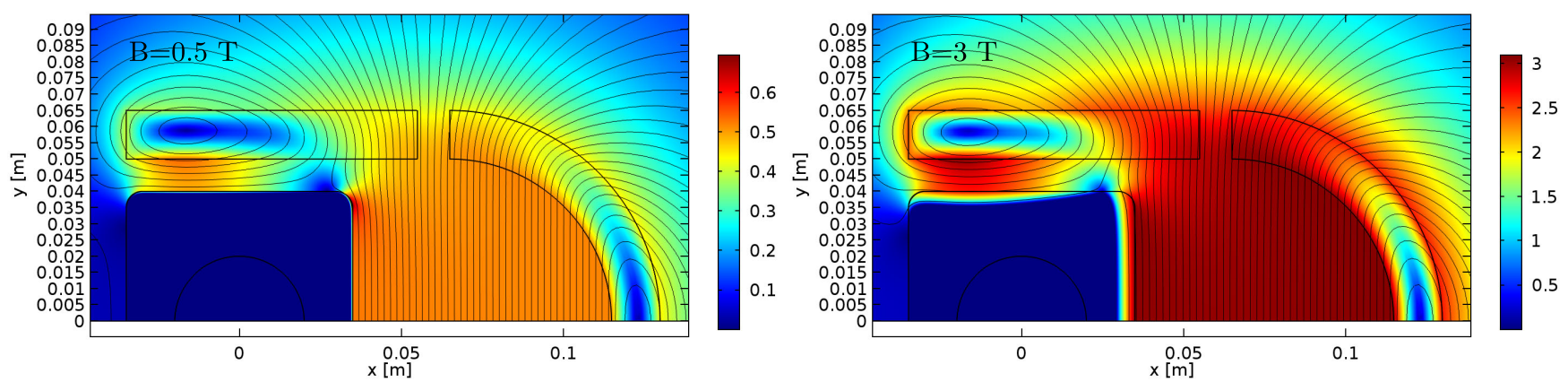

FIG. 5. Field pattern around a rectangular shield at a field strength of 0.5 and $3 \mathrm{~T}$ with a $\mathrm{NbTi} / \mathrm{Nb} / \mathrm{Cu}$ shield. Color indicates $|B|$ in Tesla. 

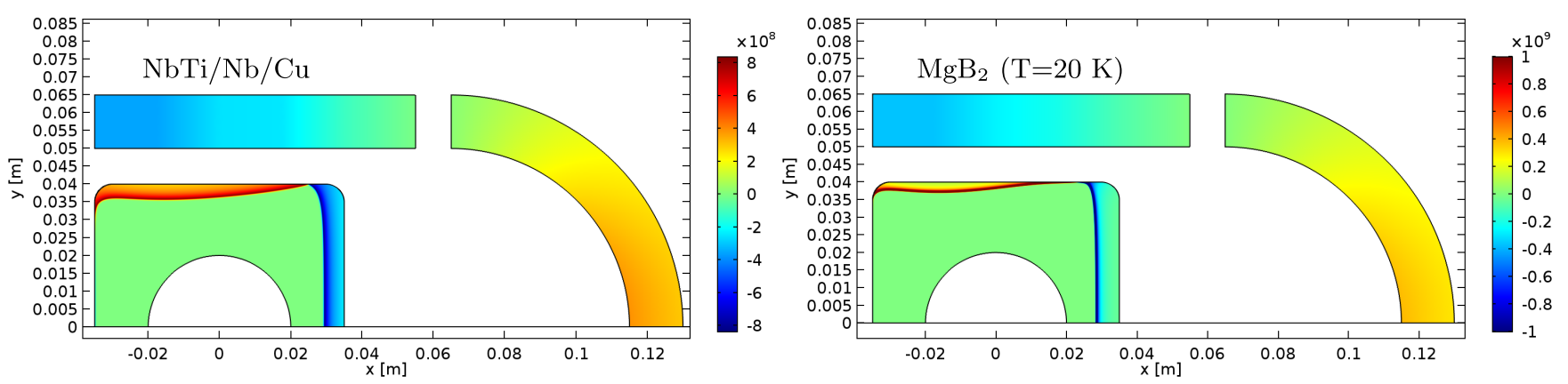

FIG. 6. Excitation and shielding current patterns assuming the $J_{c}(B)$ curve of the $\mathrm{NbTi} / \mathrm{Nb} / \mathrm{Cu}$ multilayer sheet and $\mathrm{MgB}{ }_{2}$ at $T=20 \mathrm{~K}$, at a field strength of $3 \mathrm{~T}$. Color indicates current density measured in $\mathrm{A} / \mathrm{m}^{2}$. For $\mathrm{MgB}_{2}$ the color scale was limited to $10^{9} \mathrm{~A} / \mathrm{m}^{2}$ although the highest induced current exceeds this by about a factor of 4.

the given material. Figures 7(b) and 7(c) show these numerical solutions as well (blue dashed lines) matched to the value of $B_{y}$ from the 2D FEM simulation at the surface of the shield. The agreement between the $J(x)$ and $J_{c}[B(x)]$ curves, and the $B_{\mathrm{FEM}}(x)$ and $B_{1 D}(x)$ curves is a validation of the simulation method.

The validity of these results was cross-checked by the time-dependent simulation method mentioned in Sec. IV, and a satisfactory agreement (within a few percent) was found. The homogeneity at both high and low field levels is due to the parallel displacement of the flux lines in the case of the square shield. The fact that field-independent homogeneity could be reached for both materials despite their significantly different $J_{c}(B)$ curve is very promising and indicates that the application of this concept will not be hindered by details of the material characteristics as long as their critical current density is "high enough," even though design details of a realistic device might need much more simulation studies and experimental checks. For a cylindrical shield the displacement of the flux lines at high fields is less even in space. Even though a current configuration could be found which produces an acceptable field homogeneity at low field levels, the homogeneity at high field levels could not be made better than $5 \%$.

\section{MASSLESS SEPTUM}

In the case of an asynchronous dump (when the kicker magnets fire out of synchrony with the particle-free abort gap) the beam can be swept across the septum blade. Since at the FCC the impact of even a single bunch can damage conventional materials, sophisticated protection elements are needed upstream of the septum magnets. However, the particle showers created in these elements can still reach the septum magnets and lead to heating and quenches. A massless septum (which has no material in the way of the swept beam) is insensitive to this problem; protection elements could be installed more conveniently downstream of the septa to protect the rest of the beam line, avoiding particle showers spraying the superconducting septum. Due to the symmetry of the system the shielding currents do not cross the horizontal midplane of the shield, and an opening on the shield does not intercept the path of the shielding currents.

The field pattern of a $\mathrm{NbTi} / \mathrm{Nb} / \mathrm{Cu}$ shield with the same excitation coil configuration as that shown in Fig. 5 and a massless gap of $8 \mathrm{~mm}$ was simulated for various field levels. An $8 \mathrm{~mm}$ gap seemed to be feasible to realize, and large enough to avoid large energy deposition by the beam halo during a single passage. It would nevertheless introduce a

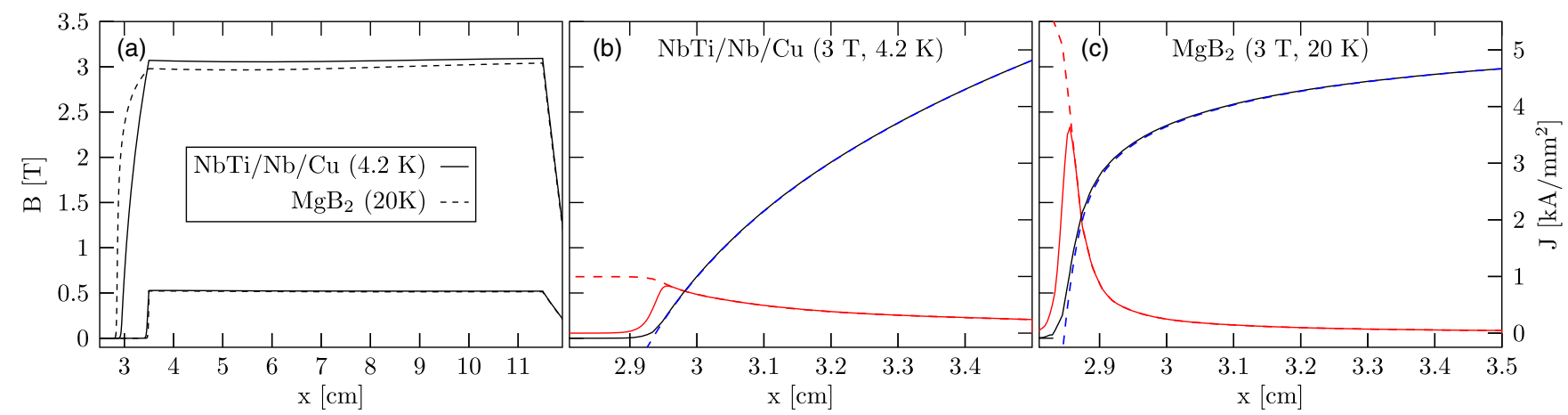

FIG. 7. (a) $B_{y}$ in the horizontal midplane for the $\mathrm{NbTi} / \mathrm{Nb} / \mathrm{Cu}$ multilayer sheet and $\mathrm{MgB}_{2}$ at $T=20 \mathrm{~K}$ at two different field levels. (b),(c) Zoom into the surface region of the shield: $B_{y}$ from the 2D FEM simulation (black solid line, left axis) and $B(x)$ from a onedimensional approximation (blue dashed line, left axis, see text for details), the shielding currents $J(x)$ (red solid line, right axis) and the critical current density at the actual level of magnetic field $J_{c}[B(x)]$ (red dashed line, right axis) for the two materials. 

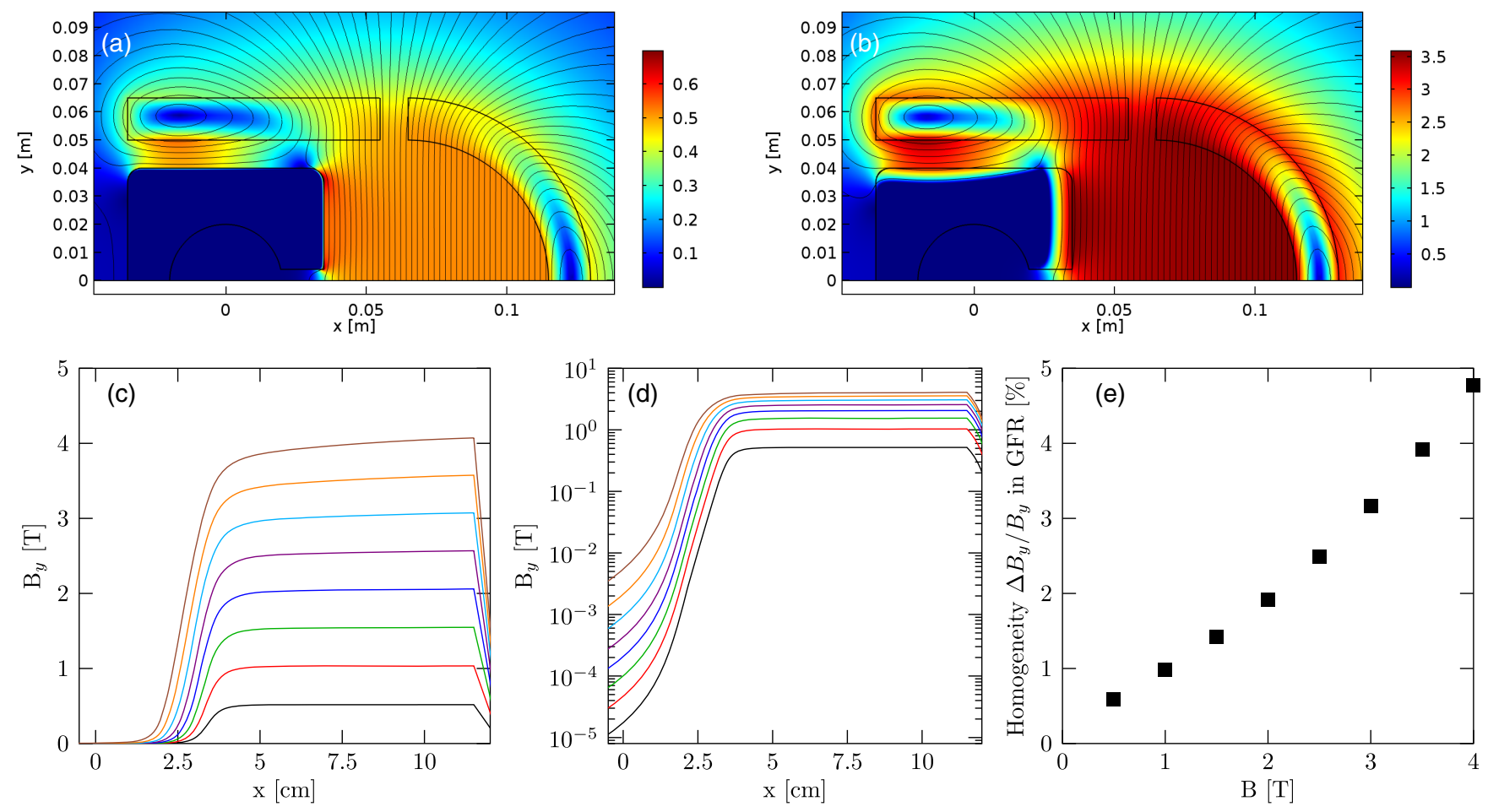

FIG. 8. (a),(b) Magnetic field pattern in a superconducting shield septum with an $8 \mathrm{~mm}$ massless gap at 0.5 and $3.5 \mathrm{~T}$. (c),(d) $B_{y}$ in the horizontal midplane on linear and logarithmic scale. (e) Homogeneity of the field in the good field region.

mechanical weakness into the shield that needs careful design to cope with. Figures 8(a)-8(d) show the field patterns and $B_{y}$ in the horizontal midplane. Field homogeneity becomes worse than $1 \%$ at field strengths larger than about $B=1 \mathrm{~T}$ as shown in Fig. 8(e).

\section{CONCLUSIONS AND OUTLOOK}

The parameters of the FCC-hh ring require a septum magnet with a magnetic field above $2 \mathrm{~T}$ for the extraction. A device using a zero-field cooled superconducting shield is proposed which creates a field-free region within a strong magnetic field via the persistent shielding currents excited in the shield by a ramped external field. Projects of the past have demonstrated that shielding 3-4 T is possible with a shield thickness that is compatible with the requirements of the FCC.

This study has addressed the quality of the field outside the shield. It is easily possible to find wire configurations producing a homogeneous field outside of a rectangular or cylindrical shield in the zero-field limit. For a rectangular shield a particular wire configuration has been found which keeps the homogeneity of the field up to $3 \mathrm{~T}$. No such wire configuration has been found for the cylindrical shield. Although this is not a proof of the nonexistence of such a wire arrangement, a shield with a rectangular outer cross section (or less restrictively, a shield with a flat wall) is favored in this application. These results have been obtained for two different $J_{c}(B)$ curves of superconductors, both of which are conservative estimates of realistic material characteristics. Higher $J_{c}$ values would lead to less deformation of the field pattern at high field levels, and improve the field independence of the homogeneity of the field pattern.

With the geometrical parameters and superconductor properties used in this study, an $8 \mathrm{~mm}$ tall massless gap in the superconducting shield does not degrade the field quality at field levels up to 1-1.5 $\mathrm{T}$, and could therefore be used for applications where this field strength is sufficient, and a massless septum is required.

A series of tests with prototypes using different materials and technologies (bulk $\mathrm{MgB}_{2}$, a multilayer $\mathrm{NbTi} / \mathrm{Nb} / \mathrm{Cu}$ sheet, and HTS tape) is planned to prove the principle and evaluate their properties. In the first series of tests a Hall probe will be installed within the shields, and the shields will be installed in the bore of a superconducting magnet, in order to test the highest field that can be shielded; the lifetime of the shielding currents; the shield's stability against thermal perturbations, flux jumps. More sophisticated measurements should then assess the effect of radiation on the shield; the highest safe warm-up and cooldown rates and the length of a reset cycle, and field homogeneity at different field strengths. One also has to develop a reliable method for the early detection of a developing quench.

As a last remark it must be noted that a superconducting shield (instead of a MuMetal shield) installed within the 
orbiting beam hole of a high-field steel-dominated Lambertson septum could solve the problem of very high leak fields [1]. This configuration is similar to the magnetic cloak mentioned earlier in the sense that the field expelled by the superconducting shield is absorbed and shaped by a ferromagnetic material, and not by the shield and the coil windings, as studied in this paper. This device would be limited to the saturation field of the used steel (typically around $2 \mathrm{~T}$ ), and the superconducting shield would be exposed to much lower field levels $(<0.5 \mathrm{~T})$ [1], allowing for a thinner shield. Exploring the properties of different superconducting shields will have a direct impact on the development of that project as well.

\section{ACKNOWLEDGMENTS}

The research leading to these results has received funding from the European Commission under the FP7 Research Infrastructures Project No. EUCARD-2, Grant Agreement No. 312453. The author would like to express his gratitude to J. Borburgh, M. Atanasov, W. Bartmann, F. Burkart, A. Sanz Ull, R. Ostojić, G. Kirby, A. Verweij, L. Bortot, A. Yamamoto, G. Grasso, G. Giunchi, and S. Molodyk for their useful help and discussions.

[1] A. Sanz Ull, High field steel dominated septa magnets, at FCC week, Rome, 2016; (private communication).

[2] F. Martin, S. Lorant, and W. Toner, A four-meter long superconducting magnetic flux exclusion tube for particle physics experiments, Nucl. Instrum. Methods 103, 503 (1972).

[3] M. Firth, E. U. Haebel, L. Krempasky, and F. Schmeissner, Performance of the superconducting field shielding tube for the CERN 2 meter hydrogen bubble chamber, in 5th Int. Conf. Instrum. High-energy Phys. edited by S. Stipcich (Laboratori Nazionali del CNEN, Frascati, 1973), pp. 79-84.

[4] M. Firth, L. Krempasky, and F. Schmeissner, Preliminary work on field-free particle beam paths from hollow superconducting shielding tubes, in Proc. Third Int. Conf. Magn. Technol. (DESY, Hamburg, 1970), p. 1178.
[5] CERN Courier, CERN 6 Tesla superconducting persistent dipole, 1979, Vol. 19, p. 109, https://cds.cern.ch/record/ 1730426/files/vol19-issue3-p109-e.pdf.

[6] A. Yamamoto et al., The superconducting inflector for the BNL g-2 experiment, Nucl. Instrum. Methods Phys. Res., Sect. A 491, 23 (2002).

[7] I. Itoh, K. Fujisawa, and H. Otsuka, NbTi/Nb/Cu Multilayer Composite Materials for Superconducting Magnetic Shielding, Nippon Steel Tech. Rep. 85, 118 (2002).

[8] R. Minet, H. Combet, J. Le Traon, and J. Schmouker, Contribution to the Use of Superconducting Shields for Magnetic Field Shaping, IEEE Trans. Magn. 3, 271 (1967).

[9] K. Sinokita, R. Toda, and Y. Sasaki, Construction of compact MRI magnet with superconducting shield, J. Phys. Conf. Ser. 150, 012041 (2009).

[10] F. Gömöry, M. Solovyov, J. Souc, C. Navau, J. PratCamps, and A. Sanchez, Experimental Realization of a Magnetic Cloak, Science 335, 1466 (2012).

[11] I. Itoh, T. Sasaki, S. Minamino, and T. Shimizu, Magnetic shielding properties of $\mathrm{NbTi} / \mathrm{Nb} / \mathrm{Cu}$ multilayer composite tubes, IEEE Trans. Appl. Supercond. 3, 177 (1993).

[12] G. Giunchi, D. Turrioni, V. Kashikhin, H. Nguyen, and E. Barzi, Feasibility study for a $\mathrm{MgB} 2$ superconducting magnetic cloak, IEEE Trans. Appl. Supercond. 26, 8801005 (2016).

[13] S. Samoilenkov, A. Molodyk, S. Lee, V. Petrykin, V. Kalitka, I. Martynova, A. Makarevich, A. Markelov, M. Moyzykh, and A. Blednov, Customised 2G HTS wire for applications, Supercond. Sci. Technol. 29, 024001 (2016).

[14] G. Giunchi, High density MgB2 obtained by reactive liquid Mg infiltration, Int. J. Mod. Phys. B 17, 453 (2003).

[15] C.P. Bean, Magnetization of hard superconductors, Phys. Rev. Lett. 8, 250 (1962).

[16] A. M. Campbell, A new method of determining the critical state in superconductors, Supercond. Sci. Technol. 20, 292 (2007).

[17] A. M. Wolsky and A. M. Campbell, A new method for determining the critical state of three-dimensional superconductors: explanation and examples, Supercond. Sci. Technol. 21, 075021 (2008).

[18] I. Itoh and T. Sasaki, Critical current density of superconducting $\mathrm{NbTi} / \mathrm{Nb} / \mathrm{Cu}$ multilayer composite sheets, Cryogenics 35, 403 (1995).

[19] G. Giunchi, The Superconducting Properties of High Density Bulk MgB2 Obtained by Reactive Liquid Mg Infiltration, in AIP Conf. Proc. 824, 813 (2006). 